\title{
Dual earthquake resistant frames
}

\author{
T. L. Sophocleous \& M. C. Phocas \\ Department of Civil and Environmental Engineering, \\ University of Cyprus, Cyprus
}

\begin{abstract}
Structural control through energy dissipation systems has been increasingly implemented internationally in recent years and has proven to be a most promising strategy for the earthquake safety of structures. In extending the "classical" approach of the capacity design for earthquake structural resistance, the integration of passive damping devices within the structure aims at energy dissipation within specific structural zones. The present paper examines an alternative control system for achieving dynamic structural adaptability, which consists of an energy dissipation device and a cable bracing mechanism with a kinetic closed circuit, working only in tension. The closed bracing mechanism does not practically affect the initial stiffness of the system, i.e. the concept relies on two completely "separate" systems: a primary for the vertical- and wind loads and a secondary for the earthquake loads. An additional feature of the bracingdamper mechanism compared to conventionally passively controlled systems is the contribution of all bracing members to the energy dissipation during a loading cycle. Three "dual systems" with different configurations of the closed bracing mechanisms and damping devices are investigated in their dynamic behaviour, in the time-history range under actual earthquakes of the GreekMediterranean region. The study provides significant response comparisons of the dual systems, in respect to the stiffness of the hysteretic dampers, its effect on the base shear force and the maximum relative displacements of the systems and to the energy dissipation behaviour of the bracing-damper mechanism.
\end{abstract}

Keywords: dual systems, hysteretic dampers, passive control.

\section{Introduction}

Form, construction and energy efficiency of the structures in earthquake prone areas depend on the integration, from an early design stage, of those structural 
elements that may optimize the earthquake systems' behaviour. Under the expected dynamic earthquake loads, an adaptable behaviour of the structure is mostly needed. The present paper examines innovative, alternative control methods for achieving dynamic adaptable structural behaviour. The attempt is to extend the design approach for earthquake resistance that is covered in most seismic codes, whereas an adaptable performance of the structures during earthquake shaking is required, in order to be able to absorb and dissipate energy in a stable manner for a large number of cycles. The seismic input energy is concentrated to specially detailed, ductile plastic hinge regions of the primary systems, where large plastic deformations can be developed. This means however severe structural damages of the respective structural elements.

The strategy of added damping for passive control is attractive for two main reasons: On one hand, damage to the gravity-load resisting frame is substantially reduced, leaving the primary frame to resist strong ground motions through exclusive elastic deformations; on the other hand, earthquake damaged dampers can be easily replaced without affecting the primary frame. For these reasons, the integration of energy dissipation devices into the primary structures provides a promising strategy, Symans et al. [1].

Plastic hysteretic dampers dissipate energy through plastic deformations of metals. Although the devices may display stable hysteresis loops under a large number of loading cycles, their integration within the main structures causes most often an increase not only of the damping but also of the stiffness of the systems, resulting to an increase of both, the base shear force and the input energy. In addition, conventionally passively controlled systems consist of braced steel frames, whereas in every half-loading cycle the compression diagonal does not participate in the energy dissipation process, since it buckles at a lower stress, than at the one needed for the damping devices to yield inelastically, Tsai et al. [2]. On the other hand, although tension-only bracings have been effectively used in some cases to resist earthquake-induced lateral forces, their inelastic cyclic hysteresis is generally poor.

In the present study cable-bracings are designed to avoid brittle failure modes, since a ductile load path is developed, whereas energy dissipation devices are designed to dissipate maximum input energy through their hysteretic behaviour. The dual structural systems, based on the concept originally proposed in Phocas and Pocanschi [3], are investigated in their earthquake responses, in three different structural configurations. The kinetics of the proposed systems reveals clearly the design concept of dual systems. The cable-bracings are fixed supported at the columns base, while connected at eccentric disks near the main frame connection joints. The cable-bracings form a closed loop in all their configurations, so that the reaction forces on the primary frame elements are neutralized. The cables follow the rotations of the eccentric discs that connect the elements with the main frame, providing a smooth transition of forces at the end of each loading cycle, when the load changes its direction. As a result, the cables are elongating in every half loading cycle, maximizing thus their yielding potential under tension and also developing a ductile load path. Tension forces are transferred, introducing an action to the optimal joint, where the hysteretic 

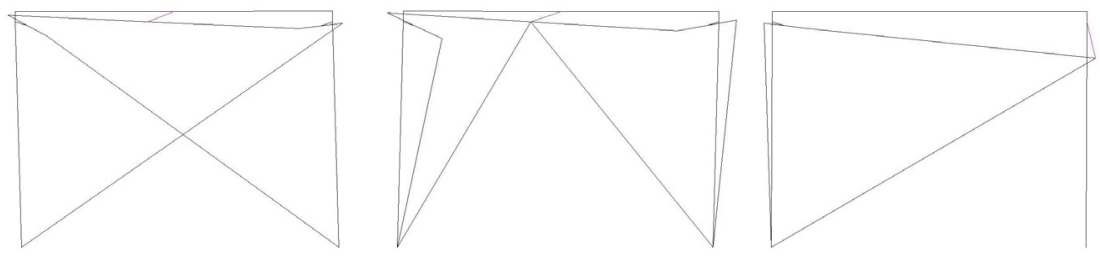

Figure 1: Deformed shapes of dual systems configurations 1, 2 and 3.

damper is placed; in the dual system 1 and 2 between the horizontal cable and the beam and in the dual system 3, between the horizontal and diagonal cable and the upper part of the column, fig. 1.

Triangular shaped steel plates take advantage of the relative movement between their ends: they utilize the relative motion of the connecting cable and the structural main frame, to yield under their inelastic deformation and dissipate a large proportion of the input energy. A major amount of input energy is dissipated, leaving the primary frame to resist the horizontal loading elastically. In a previous study, Phocas and Sophocleous [4], comparison results of the earthquake behaviour of dual system configurations and the bare frame proved the benefit of the bracing-damper control mechanism for earthquake structural safety. The present investigations seek to further verify analytically the effectiveness of the control system.

\section{Hysteretic behaviour of bracing-damper mechanism}

Hysteretic dampers dissipate energy through plastic deformations of metals. A wide variety of devices has been developed and tested that dissipate energy in flexural, shear or axial deformation modes. The hysteretic dampers used in the dual systems consist of triangular steel plates, installed in parallel rows, typically within the respective primary frame bay, between the bracing configurations and the primary frame elements. The steel dampers considered in the present study, due to their triangular shape section, develop uniform bending curvature, produced by the transverse force applying at the end of each plate, over its full height, so that all steel triangular shaped section lines reach at the same time their yielding potential. In the present study further success in a more stable hysteretic curve shape under repeated loading cycles is achieved, as shown in fig. 2 , compared to the hysteretic behaviour of the dampers used in the dual systems, investigated in Phocas and Sophocleous [4].

Defining the variables $\mathrm{t}, \mathrm{b}$ and $\mathrm{h}$ for the thickness, width, and height of a plate section respectively, and $\mathrm{n}$, for the number of damper plates, the elastic lateral stiffness of the damper is given by the following equation:

$$
\mathrm{k}_{\mathrm{d}}=\frac{\mathrm{nEbt}^{3}}{4 \mathrm{~h}^{3}}
$$



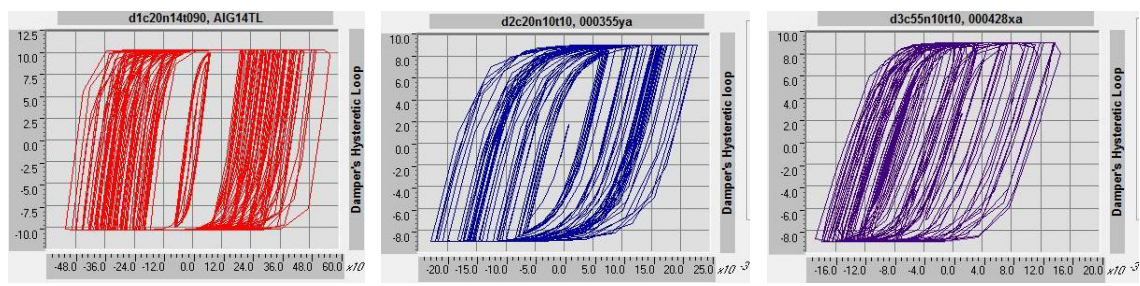

Figure 2: Hysteretic behaviour of triangular shaped steel plates, acting as plastic hysteretic dampers in dual systems 1, 2 and 3.

The plastic yield force, Py, of the device is given by

$$
\mathrm{P}_{\mathrm{y}}=\frac{\mathrm{nf}_{\mathrm{y}} \mathrm{bt}^{2}}{6 \mathrm{~h}}
$$

where fy is the yield stress $\left(\mathrm{S} 235, \mathrm{E}=2.1 \times 104 \mathrm{kN} / \mathrm{cm}^{2}\right.$, fy $=24 \mathrm{kN} / \mathrm{cm}^{2}, \rho=$ $78.5 \mathrm{kN} / \mathrm{m}^{3}$ ).

The dampers are modelled as link elements and their nonlinear behaviour as uniaxial plasticity (Wen model), whereas their nonlinear properties are assumed to follow the plastic (Wen) type, in which the force-deformation relationship involves the elastic spring constant, the yield force, the specified ratio of postyield stiffness to the elastic stiffness and an internal hysteretic variable that evolves according to a differential equation.

\section{Influence of the stiffness of the bracing-damper mechanism}

Dimensioning of the primary beam and columns was the result of a static analysis, based on Eurocode 3 guidelines. IPBv 500 section was selected for the columns and IPBv 550 section for the beam to resist elastically a vertical load of $1200 \mathrm{kN}$ and a horizontal load of $15 \mathrm{kN}$. The frame's height is $4.50 \mathrm{~m}$ and its opening $6.00 \mathrm{~m}$. The structural performance of the three dual systems was examined against the ten time varying loads of actual earthquakes of the GreekMediterranean region, with peak ground accelerations in the range from $0.10 \mathrm{~g} \div$ $0.50 \mathrm{~g}$, Phocas and Sophocleous [4]. Nonlinear time history structural analysis was conducted with the SAP2000 software program. Zero damping was considered for all excitations loading.

In the analysis the steel dampers dissipated a major part of the earthquake input energy, in dependence to the characteristics of the ground motions. Based on the results from the research study in Phocas and Pocanschi [3], initial values of a stiffness factor $\mathrm{k}$ (damper to structure stiffness), $\mathrm{k}=0.1062$, in combination with a cable's diameter of $\mathrm{dc}=20 \mathrm{~mm}$, were selected. Within the suggested value areas, the dual systems can effectively dissipate a large portion of the seismic input energy in respect to typical earthquake excitations of the GreekMediterranean region, with no significant influence on the magnitude of the base shear force that would affect the input energy in a linearly proportional function. 


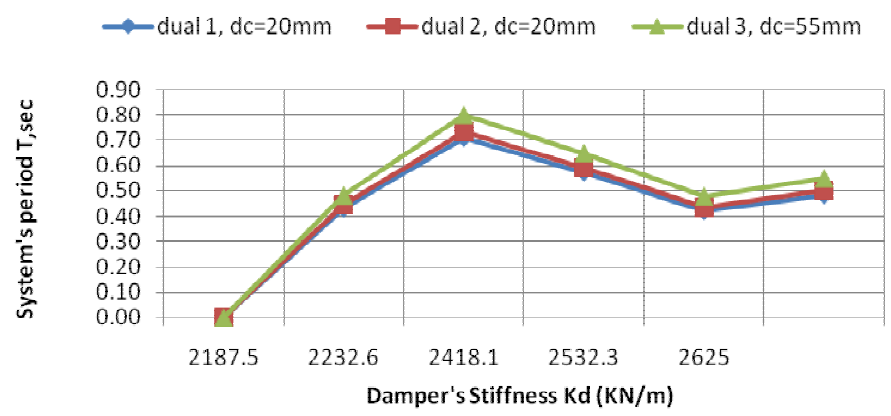

Figure 3: Relation of systems period to the dampers stiffness.

Detailed results in respect to the controlled systems' period compared to the period of $\mathrm{T}=0.36 \mathrm{sec}$ of the bare frame prove that the bracing-damper control mechanism may be attached to the primary frame, without practically increasing the systems stiffness that would also lead to an increase of the earthquake input energy, fig. 3. In addition, the primary frame may indeed be designed to resist only the gravitational and wind loads, while the control mechanism may be designed to resist the earthquake loading.

The results obtained in the time-history analyses for the resulting base shear forces prove that the dual system 3 demonstrated the best performance in resisting the Aigio 95 earthquake, for all four selected parameters in its bracingdamper design (cables and triangular shaped steel plates). At the same time, the dual system 3 seems to be the worst choice to resist the Athens 99 earthquake input. In general there are clear similarities in the earthquake responses of the dual systems 1 and 2, as shown in fig. 4. Since there is no differentiation between the selected design parameters for the damper steel plates applied in the analysis, it seems that a main reason for the third dual system's different earthquake behaviour lies on the cable's diameter, that is $\mathrm{dc}=55 \mathrm{~mm}$, instead of $\mathrm{dc}=20 \mathrm{~mm}$ used for the first two system alternatives. It may be useful to be mentioned that dual system 3 with a cable's diameter of $d c=20 \mathrm{~mm}$ did not lead to any substantial energy dissipation.

Maximum and minimum relative displacement magnitudes remained at the same levels for all three dual systems configurations (1st, 2nd and 3rd column respectively) in $40 \%$ of the earthquake inputs (Athens 99, Preveza 81 , Gulf of Corinth 93 and Ionian 83), fig. 5. Substantial differences among the dual systems behaviour could be observed under the Aigio 95 earthquake and in lower levels under the Kalamata 86, Aigio 90 and Etolia 88 earthquakes. In the latter cases, the dual system 3 proved to be the best selection, in $40 \%$ of the cases. In a single case, for the Heraklio 84 earthquake, the damper's parameters affected the response behaviour, where the dual system 3 developed the best responses for all damper's stiffness values, except for the value of $\mathrm{kd}=2418.1 \mathrm{KN} / \mathrm{m}$. In respect to the energy dissipation behaviour results, as described in the next section, no proportional relation or coupling effect is evident. 


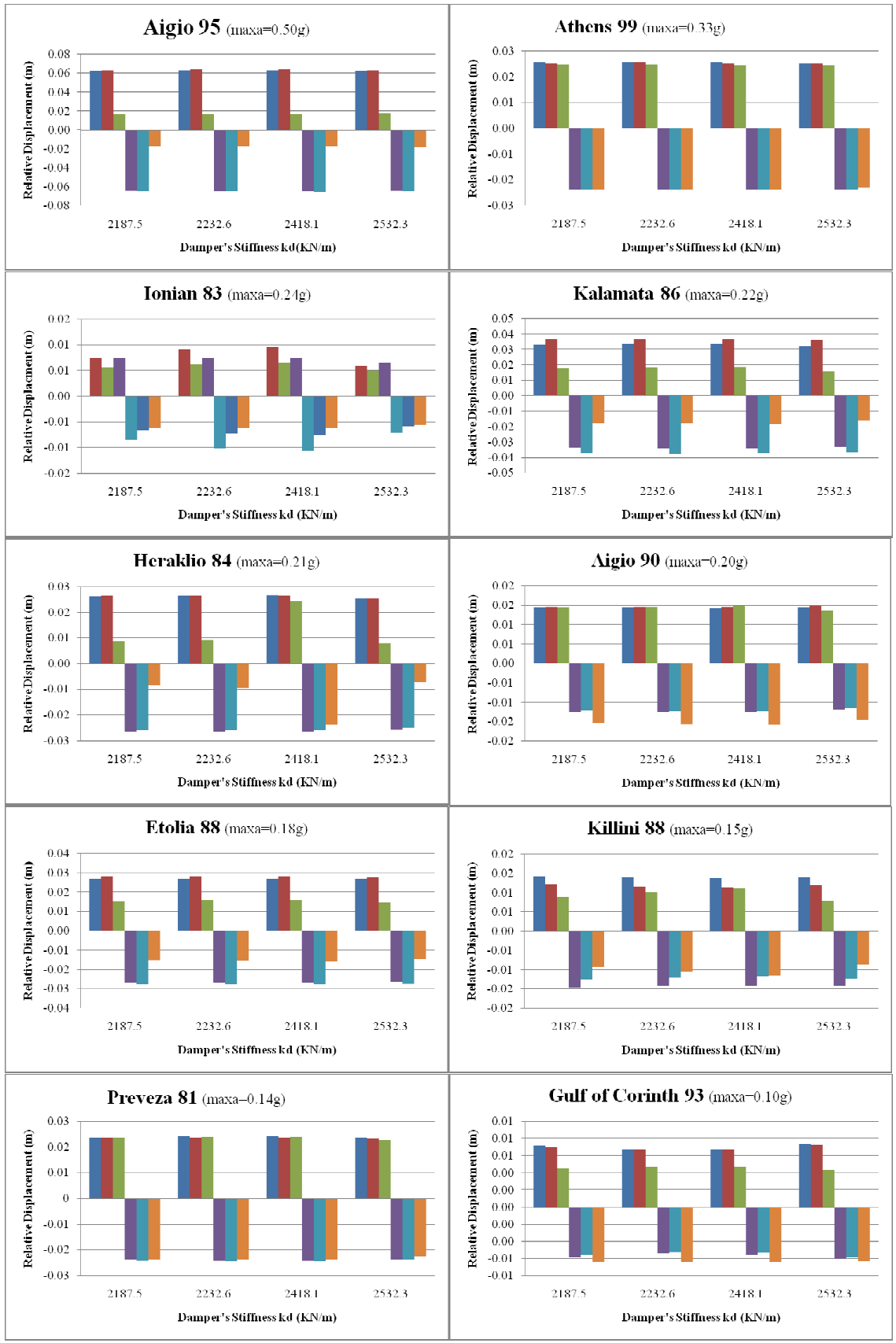

Figure 4: Relation of the resulting base shear to the dampers stiffness. 


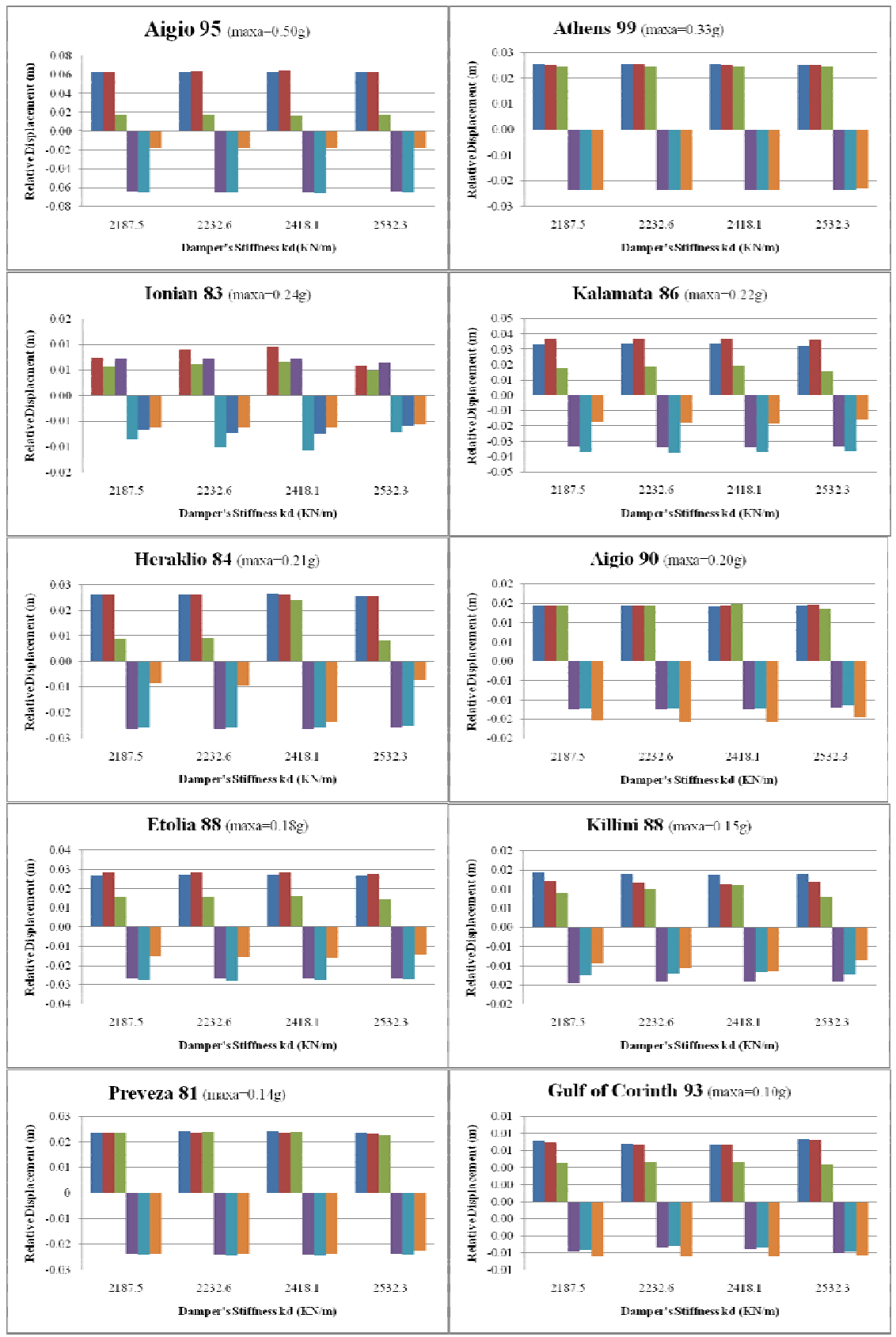

Figure 5: Relation of the resulting maximum relative displacements to the dampers stiffness. 


\section{Energy dissipation behaviour of the bracing-damper mechanism}

The analysis results indicate that in most of the cases all three systems configurations dissipated high portions of the input energy. With only one exception for the Gulf of Corinth 93 earthquake in respect to the dual system configuration 3 (3rd column), energy dissipation levels were more than satisfactory. The dual system configuration 3 performed generally better in $50 \%$ of the cases for the dampers stiffness range applied, and the dual system configuration 1 ( 1 st column) in $40 \%$ of the cases. All three dual systems have succeeded in dissipating more than $70 \%$ of the seismic energy for the earthquakes of Aigio 90, Etolia 88, Killini 88 and Preveza 81.

Energy dissipation diagrams were conducted to trace the best possible behaviour by defining and calculating a ratio between the hysteretic energy dissipated and the earthquake input energy, for different values of damper's stiffness, as shown in fig. 5.

In respect to the best results achieved, as indicated in fig. 6 , the dual system 1 dissipated $92.92 \%$ of the input energy as to the Ionian 83 earthquake. In this case, the system consisted of cables with section diameter of $\mathrm{dc}=20 \mathrm{~mm}$ and a steel damper of 10 triangular steel plates of $20 \mathrm{~cm}$ height, $5 \mathrm{~cm}$ length and 1.05 $\mathrm{cm}$ width. The dual system 2 showed the best energy dissipation behaviour for the same parameters $(\mathrm{dc}=20 \mathrm{~mm}, \mathrm{n}=10 \mathrm{~mm}, \mathrm{t}=10.5 \mathrm{~mm})$, dissipating $90.3 \%$ of the Athens 99 earthquake's input energy, whereas the dual system 3 dissipated $92.14 \%$ of the input energy induced by the same earthquake, and with a $55 \mathrm{~mm}$ cable diameter and 10 steel triangular steel plates of $10 \mathrm{~mm}$ width. In general, the dual systems configurations 1 and 2 developed very similar response characteristics.

\section{Conclusions}

In the present study, the earthquake responses of three different dual systems configurations have been investigated based on simplified analytical models of the controlled systems and ten typical earthquakes of the Greek-Mediterranean region. Opposite to the fact that in conventionally passively controlled braced frames with hysteretic dampers, the bracing components lead to an increase of the stiffness of the primary systems, dual systems' innovative mechanism proposed, aims at reducing the interactive stiffness effect on the primary frame, whereas its function relies on a continuous most uniform counteraction of all structural members to resist the earthquake loading. The present time-history analysis indicates that there is no coupling effect between the added bracingdamper mechanism and the primary frame. Therefore the application of the control mechanism becomes an attractive alternative, not only for the design of earthquake resistant structures but also for the seismic retrofit of existing ones. 


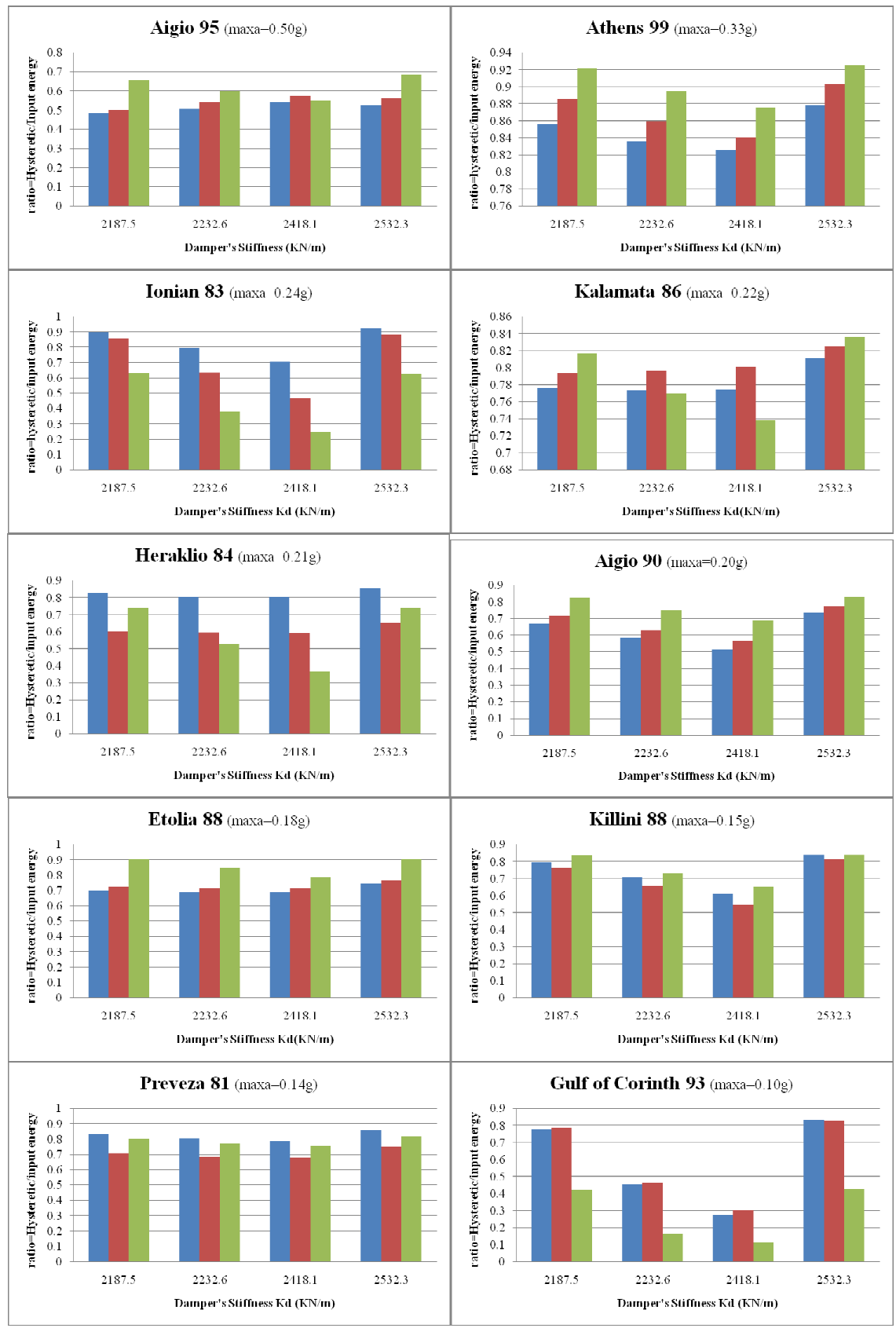

Figure 6: Relation of hysteretic dissipated energy as a portion of the input seismic energy to the dampers stiffness. 

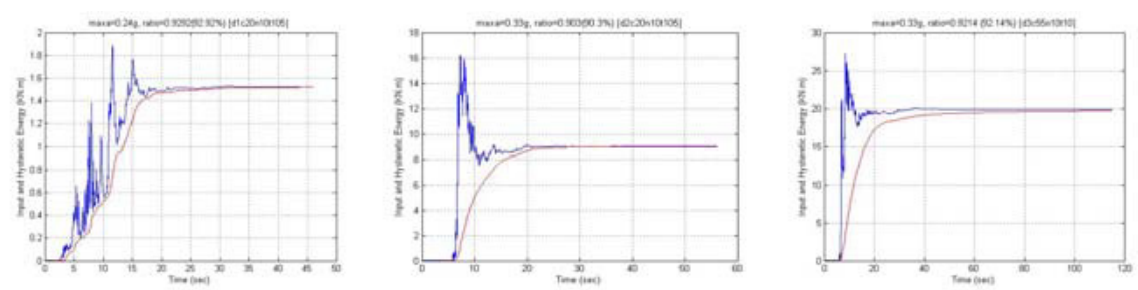

Figure 7: $\quad$ Energy dissipation behaviour of dual systems 1, 2 and 3.

The parametric study conducted for three dual systems proved that the primary structure remains to resist within the elastic region of its stress-strain diagram the horizontal forces under the earthquake induced ground motions. Besides the efficiency of the bracing-damper mechanisms to dissipate high portions of the input earthquake energy, the particular relationships between the stiffness values of the control mechanism to the primary frame proved to be critical for the desired optimum behaviour of the controlled systems. On this line, further parametric studies planned by the authors, aim at the determination of the optimum stiffness relations of the bracing-damper mechanism to the primary structure, that will enable the development of design methodologies of the dual systems according to different earthquake action types.

\section{References}

[1] Symans, M.D., Charney, F.A., Whittaker, A.S., Constantinou, M.C., Kirchner, C.A., Johnson, M.W. \& McNamara R.J., Energy dissipation systems for seismic applications: Current Practice and recent developments. Journal of Structural Engineering, 134(1), 3-21, 2008.

[2] Tsai, K.C., Chen, H.W., Hong, C.P. \& Su, Y.F., Design of steel triangular plate energy absorbers for seismic-resistant construction. Earthquake Spectra, 9(3), 502-528, 1993.

[3] Phocas, M.C. \& Pocanschi, A., Steel frames with bracing mechanism and hysteretic dampers. Journal of Earthquake Engineering and Structural Dynamics, 32, 811-825, 2003.

[4] Phocas, M.C., Sophocleous, T., Kinetic Structures in Architecture. 14th World Conference on Earthquake Engineering, 14WCEE: Beijing, China, October 2008. 